PROCEEDINGS OF THE

AMERICAN MATHEMATICAL SOCIETY

Volume 129 , Number 4 , Pages 1087-1096

S 0002-9939(00)05845-7

Article electronically published on October 4, 2000

\title{
VALIDITY OF NONLINEAR GEOMETRIC OPTICS WITH TIMES GROWING LOGARITHMICALLY
}

\author{
DAVID LANNES AND JEFFREY RAUCH
}

(Communicated by David S. Tartakoff)

\begin{abstract}
The profiles (a.k.a. amplitudes) which enter in the approximate solutions of nonlinear geometric optics satisfy equations, sometimes called the slowly varying amplitude equations, which are simpler than the original hyperbolic systems. When the underlying problem is conservative one often finds that the amplitudes are defined for all time and are uniformly bounded. The approximations of nonlinear geometric optics typically have percentage error which tends to zero uniformly on bounded time intervals as the wavelength $\epsilon$ tends to zero. Under suitable hypotheses when the amplitude is uniformly bounded in space and time we show that the percentage error tends to zero uniformly on time intervals $[0, C|\ln \epsilon|]$ which grow logarithmically. The proof relies in an essential way on the fact that one has a corrector to the leading term of geometric optics.
\end{abstract}

\section{INTRODUCTION}

The algorithms of nonlinear geometric optics construct approximate solutions of hyperbolic systems of partial differential equations. In the case of constant coefficients the operators take the form

$$
L^{\epsilon} \mathrm{u}^{\epsilon}+F\left(\mathrm{u}^{\epsilon}\right)=0 .
$$

Here $L^{\epsilon}$ is assumed to be a conservative symmetric hyperbolic system

$$
L^{\epsilon}\left(\partial_{x}\right)=L^{\epsilon}\left(\partial_{t}, \partial_{y}\right):=\frac{\partial}{\partial t}+\sum_{j=1}^{d} A_{i} \frac{\partial}{\partial y_{j}}+\frac{L_{0}}{\epsilon},
$$

in $d+1$ dimensional space time with coordinates

$$
x:=\left(x_{0}, x_{1}, \ldots, x_{d}\right)=(t, y)=\left(t, y_{1}, \ldots, y_{d}\right) .
$$

This operator satisfies

$$
L^{\epsilon}=\frac{1}{\epsilon} L\left(\epsilon \partial_{x}\right), \quad L\left(\partial_{x}\right):=\frac{\partial}{\partial t}+\sum_{j=1}^{d} A_{i} \frac{\partial}{\partial y_{j}}+L_{0} .
$$

Received by the editors June 30, 1999.

1991 Mathematics Subject Classification. Primary 35B25, 35B40, 35L60, 35Q60, 78A60, $35 \mathrm{C} 20,35 \mathrm{~F} 25$.

Key words and phrases. Nonlinear geometric optics, large time asymptotics, corrector.

This research was partially supported by the U.S. National Science Foundation, and the NSFCNRS cooperation program under grants number NSF-DMS-9500823 and NSF-INT-9314095 respectively, and the CNRS through the Groupe de Recherche G1180 POAN.

(C)2000 American Mathematical Society 
The coefficients satisfy

$$
A_{j}=A_{j}^{*} \quad \text { and } \quad L_{0}=-L_{0}^{*}
$$

which together imply the conservation law

$$
\partial_{t} \int_{\mathbb{R}^{d}}\left|u^{\epsilon}\right|^{2} d y=0
$$

for solutions of $L^{\epsilon} u^{\epsilon}=0$ which decay as $y \rightarrow \infty$.

The nonlinear function satisfies

$$
F \in C^{\infty}\left(\mathbb{C}^{N} ; \mathbb{C}^{N}\right) \quad \text { and } \quad F(0)=0 .
$$

The geometric optics ansatz yields a family of approximate solutions of the form

$$
u^{\epsilon}(x):=U_{0}(x, \beta \cdot x / \epsilon)+\epsilon U_{1}(x, \beta \cdot x / \epsilon):=U^{\epsilon}(x, \beta \cdot x / \epsilon),
$$

with

$$
\beta:=(\tau, \eta)=\left(\tau, \eta_{1}, \ldots, \eta_{d}\right) \in \mathbb{R}^{1+d} \backslash\{0\} .
$$

Assumption 1. $\beta$ is characteristic in the sense that

$$
\operatorname{det} L(i \beta):=\operatorname{det}\left(i \tau I+i \sum_{j} A_{j} \eta_{j}+L_{0}\right)=0,
$$

and either $L_{0}=0$ or $\operatorname{det}\left(\tau I+\sum_{j} A_{j} \eta_{j}\right) \neq 0$.

The profiles $U_{0}(x, \theta)$ and $U_{1}(x, \theta)$ are smooth and are $2 \pi$-periodic in $\theta$.

With $\pi(n \beta)$ defined to be the orthogonal projector onto $\operatorname{ker} L(i n \beta)$, define the operator $\Pi(\beta)$ on trigonometric series by

$$
\Pi(\beta)\left(\sum_{n} a_{n} e^{i n \theta}\right):=\sum_{n} \pi(n \beta) a_{n} e^{i n \theta} .
$$

Define partial inverses $Q(n \beta)$ by the conditions

$$
Q(n \beta) \pi(n \beta)=0 \quad \text { and } \quad Q(n \beta) L(i n \beta)=(I-\pi(n \beta)) .
$$

The operator $\mathbf{Q}(\beta)$ is defined on trigonometric series by

$$
\mathbf{Q}(\beta)\left(\sum_{n} a_{n} e^{i n \theta}\right):=\sum_{n} Q(n \beta) a_{n} e^{i n \theta} .
$$

When $L_{0}=0, Q(n \beta)=Q(\beta) / n$ for all $n \neq 0$. When $L_{0} \neq 0$, the last part of Assumption 1 implies that $Q(n \beta) L(i n \beta)=I$ for $n$ large. In both cases the operator $\mathbf{Q}$ operates on $H^{s}$ periodic functions.

In order for $\mathrm{u}^{\epsilon}$ to be an approximate solution, the profiles $U_{0}$ and $U_{1}$ are chosen as solutions of the following equations:

$$
\begin{aligned}
& \Pi(\beta) U_{0}=U_{0}, \\
& \Pi(\beta) L_{1}\left(\partial_{x}\right) \Pi(\beta) U_{0}+\Pi(\beta) F\left(U_{0}\right)=0, \\
& U_{1}=(I-\Pi(\beta)) U_{1}=-\mathbf{Q}(\beta)\left(L_{1}\left(\partial_{x}\right) U_{0}-F\left(U_{0}\right)\right) .
\end{aligned}
$$

When these equations are satisfied the profile $U^{\epsilon}$ satisfies

$$
L^{\epsilon}\left(\partial_{x}+\frac{\beta}{\epsilon} \partial_{\theta}\right) U^{\epsilon}+F\left(U^{\epsilon}\right)=\epsilon R^{\epsilon}(x, \theta),
$$


where

$$
R^{\epsilon}(x, \theta):=\left(\frac{\partial}{\partial t}+\sum_{j=1}^{d} A_{i} \frac{\partial}{\partial y_{j}}\right) U_{1}+\frac{F\left(U_{0}+\epsilon U_{1}\right)-F\left(U_{0}\right)}{\epsilon}
$$

Equation (6) implies that the approximate solution $u^{\epsilon}$ satisfies

$$
L u^{\epsilon}+F\left(u^{\epsilon}\right)=\epsilon r^{\epsilon}, \quad r^{\epsilon}(x):=R^{\epsilon}(x, \beta \cdot x / \epsilon) .
$$

For use in analysing difference quotients as above, note that Taylor's theorem implies that there is a smooth function $G$ so that

$$
F(U+W)-F(U)=G(U, W) W \quad \text { and } \quad G(U, 0)=F^{\prime}(U) .
$$

Then

$$
R^{\epsilon}=\left(\frac{\partial}{\partial t}+\sum_{j=1}^{d} A_{i} \frac{\partial}{\partial y_{j}}\right) U_{1}+G\left(U_{0}, \epsilon U_{1}\right) U_{1}
$$

The equations (3), (4) for $U_{0}$ are supplemented by the initial condition

$$
U_{0}(0, y, \theta)=u_{0}(y, \theta)=\Pi(\beta) u_{0} \in \bigcap_{s} H^{s}\left(\mathbb{R}^{d} \times \mathbb{T}\right)
$$

A version of the usual theorem of nonlinear geometric optics is the following.

Theorem 1. $i$. There is a $\left.\left.T_{*} \in\right] 0, \infty\right]$ and a unique $U_{0}$ continuous on $\left[0, T_{*}[\right.$ with values in $\bigcap_{s} H^{s}\left(\mathbb{R}^{d} \times \mathbb{T}\right)$ which satisfies (3), (4), and (10). If $T_{*}<\infty$, then $\lim _{t \nearrow T_{*}}\left\|U_{0}(t)\right\|_{L^{\infty}}=\infty$.

ii. Define a family of exact solutions $\mathrm{u}^{\epsilon}$ to (11) whose initial data are close to those of $u^{\epsilon}$ in the sense that

$$
u^{\epsilon}(0, x)-\mathrm{u}^{\epsilon}(0, y)=W^{\epsilon}(y, y \cdot \eta / \epsilon)
$$

where for all $s$

$$
\left\|W^{\epsilon}(x, \theta)\right\|_{H^{s}\left(\mathbb{R}^{d} \times \mathbb{T}\right)}=O(\epsilon) \quad \text { as } \quad \epsilon \rightarrow 0 .
$$

If $T<T_{*}$, there is an $\epsilon(T)>0$ so that for $0<\epsilon<\epsilon(T), u^{\epsilon}$ exists on $[0, T]$. In addition,

$$
\mathrm{u}^{\epsilon}(x)=\mathrm{U}^{\epsilon}\left(x, \frac{x \cdot \beta}{\epsilon}\right), \quad \text { with } \quad \mathrm{U}^{\epsilon} \in C^{0}\left([0, T] ; \bigcap_{s} H^{s}\left(\mathbb{R}^{d} \times \mathbb{T}\right)\right),
$$

and geometric optics yield a good approximation in the sense that, for all s,

$$
\sup _{t \in[0, T]}\left\|\mathrm{U}^{\epsilon}(t, y, \theta)-U_{0}(t, y, \theta)\right\|_{H^{s}\left(\mathbb{R}_{y}^{d} \times \mathbb{T}_{\theta}\right)}=O(\epsilon)
$$

In particular, for any multi-index $\alpha \in \mathbb{N}^{d+1}$

$$
\sup _{t \in[0, T]}\left\|\left(\epsilon \partial_{x}\right)^{\alpha}\left(u^{\epsilon}(t)-u^{\epsilon}(t)\right)\right\|_{L^{2}\left(\mathbb{R}^{d}\right)}=O(\epsilon) .
$$

Examples. When $W^{\epsilon}=0$, one is considering the exact solution with the same initial data as the approximate solution. A second natural choice is $\mathrm{u}^{\epsilon}(0, x)=$ $U_{0}(0, x, y . \eta / \epsilon)$ in which case $W^{\epsilon}(y, \theta)=-\epsilon U_{1}(0, y, \theta)$. 
When the profile exists globally in time, Theorem 1 implies that for any $T>0$, there is an $\epsilon(T)>0$ so that for $\epsilon<\epsilon(T)$ the solution $u^{\epsilon}$ exists for $0 \leq t \leq T$ and is given asymptotically as $\epsilon \rightarrow 0$ by the formula (2). Thus the time of existence grows without bound as $\epsilon \rightarrow 0$. Our results go in the same direction and are sharper.

If $\beta$ is a regular point of the characteristic variety, a hypothesis generically satisfied, then the equation for $U_{0}$ is a semilinear transport equation along group lines. If in addition, the nonlinear equation conserves the $L^{2}$ norm, then $\left|U_{0}\right|$ is constant along group lines. It follows that the equation for $U_{0}$ is globally solvable and that the $L^{\infty}\left(\mathbb{R}^{d} \times \mathbb{T}\right)$ norm of $U_{0}$ is independent of $t$. In this case the approximate solutions $\mathrm{u}^{\epsilon}$ are defined globally in time.

There is a rough intuition which suggests why Theorem 1 is correct and also suggests an improvement when $U_{0}$ is uniformly bounded. The residual is $O(\epsilon)$. If these errors are amplified at most exponentially and then accumulated, the effect of the residual is estimated as $O\left(\epsilon T e^{C T}\right)$. Thus for fixed $T$ the error is estimated as $O(\epsilon)$ which is the assertion of the theorem.

The accumulated error estimate $O\left(\epsilon T e^{C T}\right)$ is $o(1)$ for times $t \leq C^{\prime}|\ln \epsilon|$ provided $C C^{\prime}<1$. This suggests that the approximate solution remains a good approximation for time which grow logarithmically as $\epsilon \rightarrow 0$. Of course this expectation would not be reasonable unless the profile $U_{0}$ were globally defined. Our main result asserts that this validity on logarithmically growing intervals is correct when $U_{0}$ is uniformly bounded.

This research was stimulated by the thesis of Wagenmaker $[\mathrm{W}]$ which is the first and only result of this sort that we know. Wagenmaker studied nonlinear geometric optics for the compressible Euler equations. He proved that the presence of small regularly distributed contact discontinuities can retard by $|\ln \epsilon|$ the formation of shocks in the acoustic fields and an asymptotic description of the resulting solutions was found. The retardation was predicted by numerical experimentation in [MRS]. The results of Wagenmaker do not fall under the umbrella of the results in this article for two reasons. First, they are quasilinear, though an extension to quasilinear problems should not pose any serious obstruction. Second, the leading profile $U_{0}$ is discontinuous in $\theta$. The short wavelength perturbations contain $O(1 / \epsilon)$ contact discontinuities. We hope that by reasoning in Lagrangian coordinates, where the contacts are stationary, general results may be found which include Wagenmaker's results.

Assumption 2. The profile $U_{0} \in C^{\infty}\left(\left[0, \infty\left[; \bigcap_{s} H^{s}\left(\mathbb{R}^{d} \times \mathbb{T}\right)\right)\right.\right.$ and is uniformly bounded in the sense that

$$
\exists C, \forall t \geq 0, \quad\left\|U_{0}(t)\right\|_{L^{\infty}\left(\mathbb{R}^{d} \times \mathbb{T}\right)} \leq C .
$$

In addition, the derivatives of $U_{0}$ grow at most polynomially in the sense that for each $s \in \mathbb{Z}_{+}$there is a polynomial $q_{s}(t)$ so that

$$
\left\|U_{0}(t)\right\|_{H^{s}\left(\mathbb{R}^{d} \times \mathbb{T}\right)} \leq q_{s}(t) .
$$

Such polynomial bounds have been proved for many globally solvable nonlinear evolution equations. A typical example is given in $\S 4$.

A key estimate in the proof is that the time evolution defined by the family of linear operators

$$
\mathcal{L}^{\epsilon}:=L^{\epsilon}\left(\partial_{x}+\frac{\beta}{\epsilon} \partial_{\theta}\right)+F^{\prime}\left(U_{0}\right)
$$


have fixed exponential rate of growth for all the $H^{s}\left(\mathbb{R}_{y}^{d} \times \mathbb{T}\right)$ norms. The rates of growth differ by polynomial prefactors.

Begin with the observation that $L^{\epsilon}\left(\partial_{x}+\frac{\beta}{\epsilon} \partial_{\theta}\right)$ is a family symmetric hyperbolic system whose solutions conserve the $L^{2}\left(\mathbb{R}^{d} \times \mathbb{T}\right)$ norm. If

$$
C_{1}:=\frac{1}{2} \sup _{\left[0, \infty\left[\times \mathbb{R}^{d} \times \mathbb{T}\right.\right.}\left\|F^{\prime}\left(U_{0}\right)+F^{\prime}\left(U_{0}\right)^{*}\right\|,
$$

then, for all $\epsilon>0,0 \leq \sigma \leq t<\infty$, and solutions of $\mathcal{L}^{\epsilon} Z=0$,

$$
\|Z(t)\|_{L^{2}\left(\mathbb{R}^{d} \times \mathbb{T}\right)} \leq e^{C_{1}(t-\sigma)}\|Z(\sigma)\|_{L^{2}\left(\mathbb{R}^{d} \times \mathbb{T}\right)} .
$$

Theorem 2. Suppose that $M$ and $\underline{C}$ are constants so that for all $\epsilon>0,0 \leq \sigma \leq$ $t<\infty$, and solutions of $\mathcal{L}^{\epsilon} Z=0$,

$$
\|Z(t)\|_{L^{2}\left(\mathbb{R}^{d} \times \mathbb{T}\right)} \leq M e^{\underline{\underline{C}(t-\sigma)}}\|Z(\sigma)\|_{L^{2}\left(\mathbb{R}^{d} \times \mathbb{T}\right)} .
$$

Then for each $s \in \mathbb{Z}_{+}$there is a polynomial $p_{s}(t)$ so that for all $\epsilon>0,0 \leq \sigma \leq t<$ $\infty$, and solutions of $\mathcal{L}^{\epsilon} Z=0$,

$$
\|Z(t)\|_{H^{s}\left(\mathbb{R}^{d} \times \mathbb{T}\right)} \leq p_{s}(t-\sigma) e^{\underline{C}(t-\sigma)}\|Z(\sigma)\|_{H^{s}\left(\mathbb{R}^{d} \times \mathbb{T}\right)} .
$$

These bounds are essential in proving the next result which is the main result of the paper.

Theorem 3. If $\underline{C}$ is as in Theorem 2 and $\tilde{C}<1 /(2 \underline{C})$, then there is an $\tilde{\epsilon}>0$ so that for $0<\epsilon<\tilde{\epsilon}$, the exact solution $\mathrm{u}^{\epsilon}$ exists for $0 \leq t \leq \tilde{C}|\ln \epsilon|$. In addition,

$$
\mathrm{u}^{\epsilon}(x)=\mathrm{U}^{\epsilon}\left(x, \frac{\beta . y}{\epsilon}\right), \quad \mathrm{U}^{\epsilon} \in C^{0}\left([0, \tilde{C}|\ln \epsilon|] ; \bigcap_{s} H^{s}\left(\mathbb{R}^{d} \times \mathbb{T}\right)\right),
$$

and geometric optics provide a good approximation in the sense that for all $s$

$$
\sup _{t \in[0, \tilde{C}|\ln \epsilon|]}\left\|\mathrm{U}^{\epsilon}(t, y, \theta)-U_{0}(t, y, \theta)\right\|_{H^{s}\left(\mathbb{R}_{y}^{d} \times \mathbb{T}_{\theta}\right)}=o(\sqrt{\epsilon}) .
$$

In particular, for any multi-index $\alpha \in \mathbb{N}^{d+1}$

$$
\sup _{t \in[0, \tilde{C} \mid \ln \epsilon]]}\left\|\left(\epsilon \partial_{x}\right)^{\alpha}\left(\mathrm{u}^{\epsilon}(t)-u^{\epsilon}(t)\right)\right\|_{L^{2}\left(\mathbb{R}^{d}\right)}=o(\sqrt{\epsilon}) .
$$

Remarks. 1. Note that the approximate solution $u^{\epsilon}$ has $L^{\infty}\left(\mathbb{R}^{d}\right)$ norm which is independent of time. If the characteristic variety of $L^{\epsilon}$ consists of curved sheets, then the $L^{\infty}$ norm of the exact solution tends to zero at an algebraic rate $t^{-p}$ as $t \rightarrow \infty$. This suggests that for times which grow logarithmically, the exact solution will have decayed logarithmically and the approximate solution will not have decayed suggesting that Theorem 3 cannot possibly be correct! The error here is that as $\epsilon \rightarrow 0$ the solutions are of higher and higher frequency and are more and more columated, which heads off the decay.

2. The intuition based on decay is not entirely incorrect. For the simplest linear problems with linear phase and curved characteristic variety the approximation of geometric optics is definitely inaccurate for times $O(1 / \epsilon)$. For such times diffractive effects become important and approximations roughly of Schrödinger equation type are required (see [D, [DJMR], JMR4, [L]). Though linear problems can be well approximated for times $o(1 / \epsilon)$ the rough estimates based on accumulated error can be simply used to construct examples showing that for nonlinear problems, one cannot go beyond times which grow logarithmically in time. 
3. For problems with nonlinear phases, for example outgoing waves with convex wave fronts, the approximate solutions of geometric optics decay at the same rate as the exact solutions. Results of Godin [G] show that the nonlinear geometric optics approximations may then be accurate globally in time.

4. The results and proof extend without modification to problems which are mildly dissipative, that is, when the zero order term is of the form $L_{0} / \epsilon+B$ with $L_{0}$ as before and $B+B^{*} \leq 0$.

5. The extension of these results to quasilinear problems requires only technical modifications. The same is true of extending to the diffractive nonlinear geometric optics of [D] and DJMR. What is important is the linear phase, a constant coefficient linearized system, and the ability to construct a corrector $U_{1}$ to reduce the residual to be smaller by a power of $\epsilon$ than the exact solution. These structures are also present in the multiphase problems satisfying a genericity hypothesis (see JMR2 2) and the results extend to that context too. The method does not extend directly to problems for which geometric optics are justified without a corrector. This includes multiphase problems in dimension one and profiles almost periodic but not quasiperiodic in $x$ ([JMR1]), multiphase multi- $d$ problems not satisfying the genericity hypotheses (JMR3]), diffractive geometric optics with rectification (JMR4, [L]) and the geometric optics of pulse propagation ([A]). The attractive alternative method of Schochet [S] justifies the geometric optics approximation by a sort of Ergodic Theory argument which does not involve correctors. We suspect that this method does not yield the improvements in this paper.

\section{Proof of Theorem 2}

The proof is by induction on $s$. The case $s=0$ is true by hypothesis. Assume that the assertion is proved for $s$. We prove the case $s+1$.

For $|\alpha| \leq s+1$ apply $D_{y, \theta}^{\alpha}$ to the equation satisfied by $Z$ to find

$$
\left(\mathcal{L}^{\epsilon}+F^{\prime}\left(U_{0}\right)\right) D^{\alpha} Z+\sum_{\gamma+\mu=\alpha, \gamma \neq 0} c(\gamma, \mu) D^{\gamma}\left(F^{\prime}\left(U_{0}\right)\right) D^{\mu} Z=0 .
$$

Since $|\mu| \leq s$ the inductive hypothesis implies that

$$
\left\|D^{\mu} Z(t+\sigma)\right\|_{L^{2}} \leq\|Z(t+\sigma)\|_{H^{s}} \leq p_{s}(t) e^{\underline{\underline{C}} t}\|Z(\sigma)\|_{H^{s}} .
$$

Choosing $\underline{s}>(d+1) / 2$, Moser's inequality yields the estimate

$$
\begin{aligned}
\left\|D^{\gamma}\left(F^{\prime}\left(U_{0}\right)\right)\right\|_{L^{\infty}} & \leq\left\|D^{\gamma}\left(F^{\prime}\left(U_{0}\right)\right)\right\|_{H \underline{s}} \leq\left\|F^{\prime}\left(U_{0}\right)\right\|_{H \underline{s}+|\gamma|} \\
& \leq\left\|F^{\prime}\left(U_{0}\right)\right\|_{H \underline{s}+|\alpha|} \leq C\left(\left\|U_{0}\right\|_{L^{\infty}}\right)\left\|U_{0}\right\|_{H \underline{s}+|\alpha|} \\
& \leq \tilde{C} q_{\underline{s}+|\alpha|}(t) .
\end{aligned}
$$

Combining the last two estimates shows that

$$
\left\|\sum c(\gamma, \mu) D^{\gamma} F^{\prime}\left(U_{0}(t)\right) D^{\mu} Z(t)\right\|_{L^{2}} \leq C q_{\underline{s}+|\alpha|}(t) p_{s}(t-\sigma) e^{\underline{C}(t-\sigma)}\|Z(\sigma)\|_{H^{s}} .
$$

Duhamel's representation then yields the estimate

$$
\begin{aligned}
& \left\|D^{\alpha} Z(t+\sigma)\right\|_{L^{2}} \leq M e^{\underline{C} t}\left\|D^{\alpha} Z(\sigma)\right\|_{L^{2}} \\
& \quad+C \int_{\sigma}^{t+\sigma} M e^{\underline{C}(t+\sigma-\rho)} q_{\underline{s}+|\alpha|}(\rho) p_{s}(\rho-\sigma) e^{\underline{C}(\rho-\sigma)}\|Z(\sigma)\|_{H^{s}} d \rho .
\end{aligned}
$$

Summing over $|\alpha| \leq s+1$ completes the proof of the inductive step. 


\section{Proof of Theorem 3}

Write the exact solution $\mathrm{u}^{\epsilon}$ as a perturbation of the approximate solution $u^{\epsilon}$,

$$
\mathrm{u}^{\epsilon}=u^{\epsilon}+v^{\epsilon}
$$

with

$$
v^{\epsilon}:=V^{\epsilon}(x, \beta \cdot x / \epsilon)
$$

Then

$$
\mathrm{u}^{\epsilon}=\mathrm{U}^{\epsilon}(x, \beta \cdot x / \epsilon), \quad \mathrm{U}^{\epsilon}:=U^{\epsilon}+V^{\epsilon}=U_{0}+\epsilon U_{1}+V^{\epsilon} .
$$

The equation for $\mathrm{u}^{\epsilon}$ is

$$
L^{\epsilon}\left(\partial_{x}\right)\left(u^{\epsilon}+v^{\epsilon}\right)+F\left(u^{\epsilon}+v^{\epsilon}\right)=0 .
$$

Subtracting the equation satisfied by the approximate solution $u^{\epsilon}$ shows that this is equivalent to

$$
L^{\epsilon}\left(\partial_{x}\right) v^{\epsilon}+G\left(u^{\epsilon}+v^{\epsilon}, v^{\epsilon}\right) v^{\epsilon}=-\epsilon r^{\epsilon} .
$$

In order that $\mathrm{u}^{\epsilon}$ defined by (13) and (14) be a solution it is sufficient that $V^{\epsilon}$ satisfy

$$
L^{\epsilon}\left(\partial_{x}+\frac{\beta}{\epsilon} \partial_{\theta}\right)\left(U^{\epsilon}+V^{\epsilon}\right)+F\left(U^{\epsilon}+V^{\epsilon}\right)=0 .
$$

Equation (6) implies that

$$
L^{\epsilon}\left(\partial_{x}+\frac{\beta}{\epsilon} \partial_{\theta}\right) U^{\epsilon}+F\left(U^{\epsilon}\right)=\epsilon R^{\epsilon} .
$$

Subtracting (17) from the equation (16) shows that it is sufficient to solve

$$
L^{\epsilon}\left(\partial_{x}+\frac{\beta}{\epsilon} \partial_{\theta}\right) V^{\epsilon}+F\left(U^{\epsilon}+V^{\epsilon}\right)-F\left(U^{\epsilon}\right)=-\epsilon R^{\epsilon} .
$$

In order to attain the initial values of $\mathrm{u}^{\epsilon}$, equation (18) is supplemented by the initial condition

$$
V^{\epsilon}(0, x, \theta)=\epsilon U_{1}(0, x, \theta)+W^{\varepsilon}(x, \theta) .
$$

Then the semilinear hyperbolic equation (18) has a unique local solution

$$
V^{\epsilon} \in C\left(\left[0, T_{*}(\epsilon)\left[; \bigcap_{s} H^{s}\left(\mathbb{R}^{d} \times \mathbb{T}\right)\right) .\right.\right.
$$

If $T_{*}<\infty$, then $\lim _{t>T_{*}(\epsilon)}\left\|V^{\epsilon}(t)\right\|_{L^{\infty}}=\infty$. The representation (13), (14), (15) of $\mathrm{u}^{\epsilon}$ in terms of $V^{\epsilon}$ together with the blowup criterion for $V^{\epsilon}$, show that the interval of existence of $u^{\epsilon}$ is equal to that of $V^{\epsilon}$.

The strategy is to estimate $V^{\epsilon}$ using (18) and Theorem 2. One starts by simplifying the nonlinear term in (18). One has

$$
F\left(U^{\epsilon}+V^{\epsilon}\right)-F\left(U^{\epsilon}\right)=F\left(U_{0}+\epsilon U_{1}+V^{\epsilon}\right)-F\left(U_{0}+\epsilon U_{1}\right) .
$$

The right hand side is the sum of three terms,

$$
\begin{aligned}
\left(F\left(U_{0}+\epsilon U_{1}+V^{\epsilon}\right)-F\right. & \left.\left(U_{0}+V^{\epsilon}\right)\right) \\
& +\left(F\left(U_{0}+V^{\epsilon}\right)-F\left(U_{0}\right)\right)+\left(F\left(U_{0}\right)-F\left(U_{0}+\epsilon U_{1}\right)\right) .
\end{aligned}
$$

These in turn are equal to

$$
G\left(U_{0}+V^{\epsilon}, \epsilon U_{1}\right) \epsilon U_{1}+G\left(U_{0}, V^{\epsilon}\right) V^{\epsilon}+G\left(U_{0}+\epsilon U_{1},-\epsilon U_{1}\right)\left(-\epsilon U_{1}\right) .
$$


The first and last terms are of the form $\epsilon H\left(\epsilon, U_{0}, \epsilon U_{1}, V^{\epsilon}\right)$ where $H$ is a smooth function of its arguments with $H(\epsilon, 0,0,0)=0$. The middle term is equal to

$$
G\left(\mathcal{U}_{0}, 0\right) V^{\epsilon}+\left(G\left(\mathcal{U}_{0}, V^{\epsilon}\right)-G\left(\mathcal{U}_{0}, 0\right)\right) V^{\epsilon}=G\left(\mathcal{U}_{0}, 0\right) V^{\epsilon}+K\left(\mathcal{U}_{0}, V^{\epsilon}\right) B\left(V^{\epsilon}, V^{\epsilon}\right),
$$

where $B$ is a smooth bilinear map.

Thus equation (18) takes the form

$$
L^{\epsilon}\left(\partial_{x}+\frac{\beta}{\epsilon} \partial_{\theta}\right) V^{\epsilon}+F^{\prime}\left(U_{0}\right) V^{\epsilon}+K\left(U_{0}, V^{\epsilon}\right) B\left(V^{\epsilon}, V^{\epsilon}\right)+\epsilon H\left(\epsilon, U_{0}, U_{1}, V^{\epsilon}\right)=\epsilon R .
$$

It is important to note that the family of operators from Theorem 2 appears in the first two terms. The other terms will be treated as error terms.

Think of equation (19) as

$$
L^{\epsilon}\left(\partial_{x}+\frac{\beta}{\epsilon} \partial_{\theta}\right) V^{\epsilon}+F^{\prime}\left(\mathcal{U}_{0}\right) V^{\epsilon}=\epsilon S^{\epsilon}(t, x, \theta) .
$$

The problem is that the size of $S^{\epsilon}$ depends on $V^{\epsilon}$ which is the object we would like to estimate. However, at $t=0$, one has for each $s$

$$
\left\|V^{\epsilon}(0, x, \theta)\right\|_{H^{s}\left(\mathbb{R}^{d} \times \mathbb{T}\right)}=\left\|\epsilon \mathcal{U}_{1}(0, x, \theta)+W^{\epsilon}(x, \theta)\right\|_{H^{s}\left(\mathbb{R}^{d} \times \mathbb{T}\right)}=O(\epsilon) .
$$

Thus for $\epsilon$ small, the initial value is smaller than $\sqrt{\epsilon}$. This permits us to define $0<t_{*}(s, \epsilon)<T_{*}(\epsilon)$ by

$$
t_{*}:=\tilde{C}|\ln \epsilon| \quad \text { if } \quad\left\|V^{\epsilon}(t)\right\|_{H^{s}\left(\mathbb{R}^{d} \times \mathbb{T}\right)}<\sqrt{\epsilon} \quad \text { for } \quad 0 \leq t \leq \tilde{C}|\ln \epsilon|,
$$

and

$$
t_{*}:=\inf \left\{0<t \leq \tilde{C}|\ln \epsilon|:\left\|V^{\epsilon}(t)\right\|_{H^{s}\left(\mathbb{R}^{d} \times \mathbb{T}\right)}=\sqrt{\epsilon}\right\} \quad \text { otherwise. }
$$

We use the so-long-as method. So long as $t \leq t_{*}$ we prove an estimate for $V^{\epsilon}$ which implies that $V^{\epsilon}$ is always smaller than a quantity much smaller than $\sqrt{\epsilon}$. This allows one to prove both that $t_{*}=\tilde{C}|\ln \epsilon|$ and the error estimate in Theorem 3 .

So long as $t \leq t_{*}$, we write

$$
L^{\epsilon}\left(\partial_{x}+\frac{\beta}{\epsilon} \partial_{\theta}\right) V^{\epsilon}+F^{\prime}\left(\mathcal{U}_{0}\right) V^{\epsilon}=\epsilon S^{\epsilon}(t, x, \theta),
$$

and proceed to estimate $S^{\epsilon}$. Thanks to equation (5) and Moser's inequality

$$
\left\|U_{1}(t)\right\|_{H^{s}\left(\mathbb{R}^{d} \times \mathbb{T}\right)}=\left\|(I-\Pi(\beta)) U_{1}(t)\right\|_{H^{s}\left(\mathbb{R}^{d} \times \mathbb{T}\right)} \leq C_{s} p_{s+1}(t) .
$$

The hypothesis on $U_{0}$ together with equations (19) and (20) shows that we have the following estimate on $S^{\epsilon}$ :

$$
\left\|S^{\epsilon}(t, x, \theta)\right\|_{H^{s}\left(\mathbb{R}^{d} \times \mathbb{T}\right)} \leq C\left(\left\|U_{0}(t), \epsilon U_{1}(t), V^{\epsilon}(t)\right\|_{L^{\infty}}\right)\left\|U_{0}(t), \epsilon U_{1}(t), V^{\epsilon}(t)\right\|_{H^{s}} .
$$

The principal profile $U_{0}$ has sup norm bounded independent of $t$. The corrector has sup norm which can be estimated using Sobolev's inequality from the $H^{s}$ norm so grows at most polynomially in $t$. Thus for $t$ growing logarithmically, $\epsilon U_{1}$ is uniformly bounded in sup norm. Finally for $t \leq t_{*}, V^{\epsilon}$ is bounded in $H^{s}$ and therefore in sup norm. Thus for $t \leq t_{*}$ the prefactor in the Moser inequality estimate for $S^{\epsilon}$ is bounded and one has

$$
\left\|S^{\epsilon}(t)\right\|_{H^{s}\left(\mathbb{R}^{d} \times \mathbb{T}\right)} \leq f_{s}(t)
$$

with polynomial $f_{s}$. 
Then Theorem 2 together with Duhamel's representation shows that for $t \leq t_{*}$

$$
\begin{aligned}
\left\|V^{\epsilon}(t)\right\|_{H^{s}\left(\mathbb{R}^{d} \times \mathbb{T}\right)} & \leq p_{s}(t) e^{\underline{\underline{C}} t}\left\|V^{\epsilon}(0)\right\|_{H^{s}\left(\mathbb{R}^{d} \times \mathbb{T}\right)}+\int_{0}^{t} p_{s}(t-\sigma) e^{\underline{\underline{C}(t-\sigma)}} \epsilon f_{s}(\sigma) d \sigma \\
& \leq \epsilon e^{\underline{\underline{C}} t}\left(\tilde{p}_{s}(t)+\int_{0}^{t} p_{s}(t-\sigma) f_{s}(\sigma) d \sigma\right) \\
& \leq \epsilon e^{\underline{\underline{C}} t} \tilde{q}_{s}(t) .
\end{aligned}
$$

This implies that for $t \leq t_{*}(s, \epsilon)$,

$$
\left\|V^{\epsilon}(t)\right\|_{H^{s}\left(\mathbb{R}^{d} \times \mathbb{T}\right)} \leq \epsilon \epsilon^{-C \tilde{C}} \tilde{q}_{s}(\tilde{C}|\ln \epsilon|)=o(\sqrt{\epsilon}),
$$

since $C \tilde{C}<1 / 2$.

First fix $\underline{s}>(d+1) / 2$. Then for $\epsilon<\epsilon(\underline{s})$ and $t \leq t_{*}(\underline{s}, \epsilon)$, one has $\left\|V^{\epsilon}\right\|_{H \underline{s}\left(\mathbb{R}^{d} \times \mathbb{T}\right)} \leq$ $\sqrt{\epsilon} / 2$. The definition of $t_{*}(\underline{s}, \epsilon)$ then implies that for $\epsilon<\epsilon(\underline{s}), t_{*}(\underline{s}, \epsilon)=\tilde{C}|\ln \epsilon|$. Then by Sobolev's inequality, for $\epsilon<\epsilon(\underline{s})$ and $t \leq \tilde{C}|\ln \epsilon|$,

$$
\left\|V^{\epsilon}\right\|_{L^{\infty}\left(\mathbb{R}^{d} \times \mathbb{T}\right)} \leq C_{s}\left\|V^{\epsilon}\right\|_{H^{s}\left(\mathbb{R}^{d} \times \mathbb{T}\right)}=o(\sqrt{\epsilon}) .
$$

This estimate shows that $V^{\epsilon}$ does not explode in sup norm and therefore proves that the interval of existence $T_{*}$ for $V^{\epsilon}$ satisfies $T_{*} \geq \tilde{C}|\ln \epsilon|$. Since $\mathrm{u}^{\epsilon}$ exists at least as long as $V^{\epsilon}$; this proves the existence part of Theorem 3.

Next turn the crank one last time. For any $s \geq \underline{s}$, estimate (21) together with the definition of $t_{*}(s, \epsilon)$ implies that for $\epsilon<\epsilon(s), t_{*}(s, \epsilon)=\tilde{C}|\ln \epsilon|$. Then (21) is the error estimate required in Theorem 3 and the proof is complete.

\section{An eXAmple}

This example includes all the mathematical essentials needed in the more complicated models of the Kerr nonlinearity in optics. The equation is

$$
0=L u+i|u|^{2} u, \quad L:=\partial_{t}+\left(\begin{array}{cc}
1 & 0 \\
0 & -1
\end{array}\right) \frac{\partial}{\partial y_{1}}+\left(\begin{array}{cc}
0 & 1 \\
1 & 0
\end{array}\right) \frac{\partial}{\partial y_{2}} .
$$

For solutions of this system $\|u(t)\|_{L^{2}\left(\mathbb{R}^{d}\right)}$ is conserved. Take $\beta=(1,-1,0)$ in which case the the polarisation equation (3) asserts that the second component of $U_{0}$ vanishes, so write $U_{0}=(a(x, \theta), 0)$ with scalar valued $a(t, y, \theta)$. The profile equation (4) is then

$$
\left(\partial_{t}+\partial_{y_{1}}\right) a+i|a|^{2} a=0 .
$$

If $a_{0}(y, \theta)$ is the initial value of $a$, one has the explicit solution

$$
a\left(t, y_{1}, y_{2}, \theta\right)=e^{i t\left|a_{0}\left(y_{1}-t, y_{2}, \theta\right)\right|^{2}} a_{0}\left(y_{1}-t, y_{2}, \theta\right) .
$$

In particular the $L^{\infty}$ norm of $a$ is constant. In fact the modulus of $a$ is constant on integral curves of the vector field $\partial_{t}+\partial_{y_{1}}$. Differentiating the solution one finds that

$$
\begin{array}{r}
D_{y, \theta} a\left(t, y_{1}, y_{2}, \theta\right)=e^{i t\left|a_{0}\left(y_{1}-t, y_{2}, \theta\right)\right|^{2}}\left(D_{y, \theta} a_{0}\left(y_{1}-t, y_{2}, \theta\right)\right. \\
\left.+i t D_{y, \theta}\left(\left|a_{0}\left(y_{1}-t, y_{2}, \theta\right)\right|^{2}\right) a_{0}\left(y_{1}-t, y_{2}, \theta\right)\right) .
\end{array}
$$

Therefore the $H^{1}$ norm grows linearly in time. A simple induction shows that the derivative of order $s$ grows at most like $t^{s}$ so the polynomial growth hypothesis is 
satisfied. One concludes that on time intervals growing logarithmically in time, the approximate solution

$$
u_{\text {approx }}^{\epsilon}:=\left(a_{0}\left(t, y,\left(t-y_{1}\right) / \epsilon\right), 0\right)
$$

differs by $o(\sqrt{\epsilon})$ from the exact solution which has the same initial data.

\section{ACKNOWLEDGEMENTS}

The authors are pleased to acknowledge the contributions of stimulating discussions of these questions with A. Binzer, O. Gues, J.-L. Joly and G. Métivier. This research was begun in November 1998 at the Université de Nice while the second author was Professeur Invité. We thank that university and our colleagues there for their generous hospitality.

\section{REFERENCES}

[A] D. Alterman, Diffractive nonlinear geometric optics for short pulses, Ph.D. Thesis, University of Michigan, 1999.

[D] P. Donnat, Quelques contributions mathématiques en optique non linéaire, Thèse Doctorale, Ecole Polytechnique, 1994.

[DJMR] P. Donnat, J.-L. Joly, G. Métivier, and J. Rauch, Diffractive nonlinear geometric optics I, Séminaire Equations aux Dérivées Partielles, Ecole Polytechnique, Palaiseau, 1995-1996, XVII1-XVII 23. MR 99m:35147

[G] P. Godin, Global oscillatory waves for second order quasilinear wave equations, Trans. Amer. Math. Soc., 346(2)(1994), 523-547. MR 95f:35157

[JMR1] J.-L. Joly, G. Métivier, and J. Rauch, Resonant one dimensional nonlinear geometric optics, J. Functional Analysis, Vol. 114(1993), 106-231. MR 94i:35118

[JMR2] J.-L. Joly, G. Métivier, and J. Rauch, Generic rigorous asymptotic expansions for weakly nonlinear multidimensional oscillatory waves, Duke Math J., 70(1993), 373-404. MR 94c:35048

[JMR3] J.-L. Joly, G. Métivier, and J. Rauch, Coherent and focusing multidimensional nonlinear geometric optics, Annales de L'École Normale Supérieur, 28(1995), 59-113. MR 95k:35035

[JMR4] J.-L. Joly, G. Métivier, and J. Rauch, Diffractive nonlinear geometric optics with rectification, Indiana U. Math. J. 47(1998), 1167-1242. MR 2000d:35130

[L] D. Lannes, Dispersive effects for nonlinear geometrical optics with rectification, Asymptotic Analysis 18 (1998) 111-146. MR 99m:35142

[MRS] A. Majda, R. Rosales, and M. Schonbek, A canonical system of integro-differential equations arising in resonant nonlinear acoustics, Stud. Appl. Math. 79(1988), 205-262. MR 90g:76077

[S] S. Schochet, Fast singular limits of hyperbolic partial differential equations, J.Diff.Eq. 114 (1994), pp. 474-512. MR 95k:35131

[W] T. Wagenmaker, Analytic solutions and resonant solutions of hyperbolic partial differential equations, Ph.D Thesis, University of Michigan, 1994.

MAB, Université de Bordeaux I, 33405 Talence, France

E-mail address: lannes@math.u-bordeaux.fr

Department of Mathematics, University of Michigan, Ann Arbor, Michigan 48109

E-mail address: rauch@math.1sa.umich.edu 